\title{
El impacto del cambio organizacional en las empresas metalmecánicas de San Juan de Río, las cuales cuentan con Sistema de Gestión de Calidad preferentemente certificado
}

\section{The impact of organizational change in the metalworking companies of San Juan de Rio, which have a Quality Management System preferably certified}

TORRES-TELLO, Marco Antonio, VILLARREAL-ALCALDE, Jorge Gabriel, CHACÓN-PENA, Jair De Jesús y URQUIZA-RESÉNDIZ, Rafael

Universidad Autónoma de Querétaro, Campus San Juan del Río, Av. Río Moctezuma No 249, Col. San Cayetan, San Juan del Río, Qro. CP: 76807

ID $1^{\text {er }}$ Autor: Marco A, Torres-Tello / ORC ID: 0000-0002-6362-6874, Researcher ID Thomson: X-3313-2018, CVU CONACYT ID: 1012398

ID $1^{\mathrm{er}}$ Coautor: Jorge, G, Villarreal-Alcalde / ORC ID: 0000-0003-3101-3683, Researcher ID Thomson: X-3222-2018, CVU CONACYT ID: 999689

\author{
ID $2^{\text {do }}$ Coautor: Jair, Chacon-Pena / ORC ID: 0000-0002-2769-9881, CVU CONACYT ID: 733695 \\ ID $3^{\text {er }}$ Coautor: Rafael, Urquiza-Reséndiz / ORC ID: 0000-0001-7456-9361, CVU CONACYT ID: 699960
}

DOI: $10.35429 / J E D .2019 .21 .6 .1 .9$

Recibido: 10 de Septiembre 2019; Aceptado 30 de Diciembre, 2019

\section{Resumen}

Hoy en día la implementación y mantenimiento de sistemas, incluyendo los de gestión de calidad coadyuvan a las empresas a desarrollarse a niveles adecuados, de tal manera que puedan integrarse a los mercados globales y poder competir de manera efectiva. En relación a esto los sistemas de calidad basados en normas internacionales, como los de ISO "International Standard Organization" han sido las más difundidas, adoptadas y aceptadas en empresas de servicios, manufactura y otros sectores. Sin embargo, esto no garantiza el éxito, las estrategias de sistemas de gestión de calidad han generado algunas dudas como su eficacia (Gryna, F. et al. 2007), donde los procesos juegan un papel importante. Con esta intención hecha operativa, los aspectos de procesos, sistemas y cambio organizacional, tanto técnico como administrativo, permiten a medir el grado de adecuación entre los procesos y su impacto al cambio positivo que contribuye a la competitividad. Esta investigación cuantitativa de una muestra de micro empresas metalmecánicas del municipio de San Juan del Río, que cuentan con sistemas de gestión de la calidad de preferencia certificados, se han contrastado las proposiciones que indagan en la correlación que existe entre la gestión de la calidad y el cambio organizacional como elementos de soporte en la competitividad.

Sistema de Gestión de Calidad, Cambio organizacional, Empresas Metalmecánica

\section{Abstract}

Today, the implementation and maintenance of systems, including those of quality management, help companies to develop at appropriate levels, so that they can integrate into global markets and be able to compete effectively. In relation to this, quality systems based on international standards, such as those of ISO "International Standard Organization" have been the most widespread, adopted and accepted in service companies, manufacturing and other sectors. However, this does not guarantee success, quality management systems strategies have generated some doubts such as their effectiveness (Gryna, F. et al., 2007), where processes play an important role. With this intention made operational, the aspects of processes, systems and organizational change, both technical and administrative, allow to measure the degree of adequacy between the processes and their impact on the positive change that contributes to competitiveness. This quantitative investigation of a sample of micromechanical micro enterprises of the municipality of San Juan del Río, which have certified quality management systems, have proven the proposals that investigate the correlation between quality management and organizational change as support elements in competitiveness.

Quality Management system, organization change, metalwork organizations

Citación: TORRES-TELLO, Marco Antonio, VILLARREAL-ALCALDE, Jorge Gabriel, CHACÓN-PENA, Jair De Jesús y URQUIZARESÉNDIZ, Rafael. El impacto del cambio organizacional en las empresas metalmecánicas de San Juan de Río, las cuales cuentan con Sistema de Gestión de Calidad preferentemente certificado. Revista de Desarrollo Económico. 2019. 6-21: 1-9

$\dagger$ Investigador contribuyendo como primer autor. 


\section{Introducción}

En esta investigación se toma en cuenta a la clasificación de empresas micro metalmecánicas en el municipio de San Juan del Rio, Querétaro, de las cuales actualmente no se cuenta con suficiente información para poder explicar la relación entre los sistemas de calidad, procesos y cambio organizacional. La nueva forma de administración en las organizaciones marco un paradigma que se fundaba en la aplicación práctica de los principios Tayloristas burocráticos que se relacionaban con el modelo de organización moderna, cabe mencionar que este tipo de organizaciones no necesariamente comparten el mismo tipo de problema o de fines, así como tampoco tienen el mismo nivel de conocimiento y de acuerdo a estos es el impacto que se presenta en la estructura organizacional.

De acuerdo a datos de la SEDESU (2015), durante el 2015 el estado de Querétaro presento un crecimiento de $7.2 \%$ del PIB del cual el $44 \%$ corresponde al sector secundario donde se incluye el manufacturero, Siendo este sector de suma relevancia para el estado, dado que el PIB estatal es fuertemente impactado por la actividad industrial.

La producción en San Juan del Río y principalmente en la ciudad de Querétaro se remonta a varias décadas atrás, Las zonas cercanas a las ciudades se han tenido que adaptar a los nuevos requerimientos de las organizaciones para aprovechar estas condiciones ser empresas más competitivas.

\section{Justificación}

La organización tiene sus inicios desde los orígenes del hombre, ya que estos se dieron cuenta que al formar grupos podían afrontar más fácilmente las inclemencias del tiempo, la caza de animales para la supervivencia e incluso enfrentar a otros grupos de humanos. Las primeras organizaciones se formaron de manera empírica, con los integrantes de las familias, después se estableció el Sistema que Marx denomino Feudal y a mediados del siglo XIX se crearon las naciones, (Hernández, S.,2006).

En la actualidad este tipo de organizaciones ya no son suficientes ante los retos de la globalización por eso ahora ya existen organizaciones más grandes.
Aunque existen diferentes tipos de organizaciones como las políticas o sociales en este estudio se enfocara a las organizaciones del sector micro manufacturero metalmecánica del municipio de San Juan del Río. La organización es un "Proceso estructurado en el cual interactúan las personas para alcanzar sus resultados" según Auridac (1995: p.31). La Norma ISO 9000 (2015) la define como un conjunto de personas e instalaciones con una disposición de responsabilidades, autoridades y relaciones para un fin o propósito. Daft (2010) afirma que "Organización es el desarrollo de recursos organizacionales para lograr metas estratégicas".

Dentro del proceso administrativo la Organización es la segunda fase, a través de ella el sistema establece la división del trabajo y la estructura necesaria para su funcionamiento; además, es una de las funciones que se ocupa de dotar a las empresas de una estructura de autoridad, responsabilidad, niveles jerárquicos, funciones, líneas de mando y comunicación.

El punto de partida para la organización es la planeación y fortalecimiento de la cultura organizacional formal, es decir haber determinado la misión, visión, filosofía, estrategias y objetivos de la empresa, los cuales son definidos por la alta dirección de la organización, esto está recomendado por las normas internacionales de Sistema de gestión de calidad como la ISO 9001 (2008) y actualmente ya liberada ISO 9001 (2015), esto llevara a poder diseñar una estructura que le permita alcanzar la misión para la que fue creada.

Uno de los principales objetivos de la actividad empresarial es lograr la rentabilidad como resultado de las actividades que realiza, es decir que su acción diaria coadyuve para que se obtengan los ingresos y utilidades planeados.

Existen numerosas normas internacionales y nacionales que tratan de ordenar y sistematizar la implantación de sistemas de gestión de calidad empresarial (ISO 9000, IATF 16949, VDA, etc.). Todas estas normas cuentan con una estructura similar, procesos de implantación, verificación, mejora continua y buscan como enfoque fundamental la satisfacción del cliente; así como lograr que los procesos dentro de la organización sean eficaces y logren los objetivos y resultados planeados para cada proceso. 
Las organizaciones en la actualidad requieren de sostener cambios constantes para adaptarse a la globalización, competencia y así poder mantenerse en los mercados. Las estrategias que estén bien diseñadas y controladas pueden ser una muy buena herramienta para facilitar los cambios en las organizaciones, y para que estos no sean tan espontáneos se requieren métodos o procesos que los inicien, controlen y faciliten, de tal manera que una opción, es aprovechar los sistemas de gestión de calidad con los que ya muchas organizaciones cuentan o están familiarizados.

Para Stewart (1992) el cambio organizacional es la adaptación de las organizaciones a diversas transformaciones que pueden sufrir tanto en su ambiente interno como en su entorno, aprendiendo de las implementaciones que busca esta adaptación.

Audirac (2011) señala que existen consideraciones a tomar en cuenta para el cambio en las organizaciones, entre ellas:

\section{"[...] Todo cambio que ocurre en un subsistema de la organización afecta a su totalidad; el cambio es un reto tanto humano como técnico ya que estos dos componentes contribuyen a dichos cambios; los cambios en las organizaciones pueden parecer injustificados cuando las personas no cuentan con los elementos para entender que sus beneficios compensan sus costos [...]" (p.83).}

La búsqueda continua de las organizaciones por la competitividad las lleva a asumirse dispuestas al cambio incesante; y para poder lograrlo como parte de esta búsqueda los directivos de empresas buscan modelos que les permitan tener proceso sistemáticos para lograr la calidad y competitividad, es por ello que como parte de este cometido se busca la certificación de gestión de calidad, como por ejemplo: la norma ISO 9001:2015 que es de aplicación general y que entre otros principios se basa en la calidad, enfoque del procesos, pensamiento basado en riesgos y la mejora continua, esta última fuertemente ligada a lograr cambios favorables.

\section{Problema}

San Juan del Río después de la capital es el municipio y ciudad más poblada del estado de Querétaro, además, de ser uno de los más productivos ya que cuenta con una solida infraestructura de apoyo para el estado. En el sector secundario se planea consolidarlo como polo industrial a través de la integración en sectores de la economía y crecimiento de empresas de alta tecnología que le permite ser una región de atracción e inversión.

Además, que por su ubicación geográfica la ciudad de San Juan del Río puede ser un punto estratégico para el desarrollo económico regional y nacional.

El crecimiento en San Juan del Río se puede explicar en gran medida por su industria, debido a esta actividad y la de su comercio que representa un 20\% del PIB total del estado de Querétaro (Periódico Rotativo 16 de marzo de 2016). Además, forma parte del "Corredor Industrial del Bajío" que va desde San Juan del Río, Querétaro, ciudades de Guanajuato hasta llegar a Guadalajara.

Debido al crecimiento que el sector industrial ha tenido en la localidad resulta interesante realizar la investigación en empresas micro y pequeñas metalmecánicas en San Juan del Río. Se busca además que estas empresas cuenten con una certificación vigente en ISO 9001:2015 o que estén en vías de obtenerla, así podrán confirmar y darse cuenta de cómo la implementación de sistemas de gestión de calidad influye y producen un cambio organizacional.

\section{Hipótesis}

Esta implica la formulación de explicaciones sujetas a comprobación o la definición de solución a situaciones.

- Existe correlación positiva entre el cambio organizacional y los sistemas de gestión de calidad.

- Las organizaciones que tienen procesos eficaces generan mayor cantidad de cambios organizacionales.

Algunos procesos basados en normas del sistema de gestión de calidad ISO 9001 tienen mayor impacto en el cambio organizacional. 
Empresas certificadas en ISO 9001 tienen mejor desempeño que las no certificadas.

\section{Objetivos}

\section{Objetivo General}

Explicar si los procesos que conforman el sistema de gestión de calidad impactan en el cambio organizacional para las organizaciones micro y pequeñas metalmecánicas en el municipio de San Juan del Río y las posibles diferencias entre empresas certificadas y no certificadas para logra dichos cambios.

Además, se pretende identificar algunas pistas importantes que nos permitan entender como la calidad puede influir de manera positiva en la organización y como los sistemas de gestión de calidad basados en normas ISO 9001 afectan $\mathrm{o}$ inciden en los cambios organizacionales.

\section{Objetivos específicos}

Identificar posibles diferencias en empresas certificadas y no certificadas en la percepción de cambio organizacional como resultado de la actividad en los procesos de los sistemas de gestión de calidad.

Analizar la interrelación entre variables de cambio organizacional y los sistemas de gestión de calidad para apoyar la implementación controlada de dichos cambios.

\section{Marco Teórico}

Aquí se estudian las distintas interpretaciones sobre los modelos organizacionales y el análisis de calidad, los sistemas de gestión de calidad y su evolución con relación a las formas organizacionales y su entorno, pasando por un análisis de las propuestas de la calidad enfocada básicamente a la producción, la relación particularmente entre la calidad, la perspectiva de la administración, su origen en Estados Unidos y su desarrollo en Japón, así como los conceptos básicos en las propuestas recientes. También se presenta un apartado que contiene la metodología y el desarrollo de la investigación en las empresas manufactureras del municipio señalado.
La calidad es sumamente importante para las organizaciones, de ella depende en gran medida la satisfacción e insatisfacción del cliente.

Las definiciones del término calidad han sido propuestas por expertos en la materia. La tabla siguiente muestra algunas de las definiciones de este campo.

\begin{tabular}{|c|c|}
\hline Autor & Definición \\
\hline $\begin{array}{l}\text { Crosby } \\
(1989)\end{array}$ & Cumplimiento de especificaciones dadas. \\
\hline $\begin{array}{l}\text { Deming } \\
(1989)\end{array}$ & $\begin{array}{l}\text { Un grado predecible de uniformidad y } \\
\text { fiabilidad a bajo costo, adecuado a } \\
\text { necesidades de mercado. }\end{array}$ \\
\hline $\begin{array}{l}\text { Drucker } \\
(1992)\end{array}$ & $\begin{array}{l}\text { Lo que el cliente está dispuesto a pagar en } \\
\text { función de lo que obtiene y valora. }\end{array}$ \\
\hline $\begin{array}{l}\text { Feigenbaum } \\
(1986)\end{array}$ & $\begin{array}{l}\text { Un sistema eficaz para integrar los } \\
\text { esfuerzos de mejora de la calidad de los } \\
\text { distintos grupos de una organización, para } \\
\text { proporcionar productos y servicios a } \\
\text { niveles que permitan la satisfacción del } \\
\text { cliente. }\end{array}$ \\
\hline $\begin{array}{ll}\text { ISO } & 9000 \\
(2005) & \end{array}$ & $\begin{array}{l}\text { Grado en el que un conjunto de } \\
\text { características inherentes cumple con los } \\
\text { requisitos. }\end{array}$ \\
\hline Juran (1990) & $\begin{array}{l}\text { Adecuación para el uso, satisfaciendo las } \\
\text { necesidades del cliente. }\end{array}$ \\
\hline $\begin{array}{l}\text { Taguchi } \\
(1979)\end{array}$ & $\begin{array}{l}\text { Perdidas mínimas para la sociedad } \\
\text { durante la vida del producto. }\end{array}$ \\
\hline $\begin{array}{ll}\text { ISO } & 9000 \\
(2005) & \end{array}$ & $\begin{array}{l}\text { Grado en el que un conjunto de } \\
\text { características inherentes cumple con los } \\
\text { requisitos }\end{array}$ \\
\hline $\begin{array}{ll}\text { ISO } & 9000 \\
(2015) & \end{array}$ & $\begin{array}{l}\text { Grado en el que un conjunto de } \\
\text { características inherentes de un objeto } \\
\text { cumple con los requisitos. }\end{array}$ \\
\hline
\end{tabular}

Tabla 1 Definiciones sobre calidad

Fuente: Elaboración propia a partir de Danvila. I y Sastre, Miguel. (2007)

Es de destacar que, aunque existen varias definiciones de calidad, todas ellas coinciden en la necesidad de cumplir requisitos explícitos e implícitos para alcanzar la satisfacción del cliente cubriendo sus necesidades y expectativas.

De acuerdo a Jun et al. (1998) se sabe que la calidad del producto o servicio es parte fundamental para el éxito de la organización. La calidad usualmente aumenta las ganancias reduciendo los costos y mejorando la posición competitiva de la firma, esto puede incidir en un impacto favorable en el cambio organizacional.

Los sistemas de gestión calidad de acuerdo a su definición con el vocabulario de ISO 9000 (2015) son: 
- Gestión, actividades coordinadas para dirigir y controlar una organización.

- Sistemas de gestión, es un conjunto de elementos mutuamente relacionados o que interactúan para establecer la política y los objetivos.

Sistema de gestión de la calidad, es un sistema de gestión para dirigir y controlar una organización con respecto a la calidad.

"La dirección y control, en lo relativo a la calidad, generalmente incluye el establecimiento de la política de la calidad y los objetivos de la calidad, la planificación de

la calidad, el control de la calidad, el aseguramiento de la calidad y la mejora de la calidad" (p.10).

La política de la calidad son las intenciones globales y orientación de una organización relativa a la calidad tal como se expresan formalmente por la alta dirección.

Los objetivos de la calidad son los aspectos ambicionados o pretendidos relacionados con la calidad.

La planeación de la calidad está enfocada al establecimiento de los objetivos de la calidad.

El control de calidad se refiere a parte de la gestión de la calidad orientada al cumplimiento de los requisitos de la calidad y el aseguramiento de la calidad proporciona confianza en que se cumplirán los requisitos de calidad.

La mejora de la calidad es una parte de la gestión de la calidad orientada a aumentar la capacidad de cumplir con los requisitos de la calidad y la mejora continua son aquellas actividades recurrentes para aumentar la capacidad para cumplir los requisitos.

De acuerdo con estas definiciones y lo declarado en la serie de normas de la familia ISO 9000, se entiende que dichas normas de sistemas de gestión de calidad son de aplicación general y se puede adaptar a cualquier tipo de organización, ya sea que desee o no su certificación.
Actualmente existe un gran número de normas internacionales y nacionales que tratan de ordenar y sistematizar la implantación de sistemas de gestión empresarial en relación a actividades operativas muy diversas como la mejora de la calidad (normas ISO 9000, ISO/TS 16949, EAQF, VDA, etc.), el impacto medioambiental (ISO 14000 y EMAS), la prevención de riesgos laborales (OHSAS 18001), o la responsabilidad social corporativa (SA 8000, AA 1000 o los proyectos de norma ISO CSR o UNE 165010). De todas estas normas destacan las normas ISO 9001. Con la proliferación de estos estándares, crece la oferta de implantación de estos sistemas por parte de las consultoras.

De acuerdo con la norma ISO 9004 (2009), se han identificado ocho principios de gestión de la calidad los cuales deben ser usado por la alta dirección con el fin de conducir a la organización a un mejor desempeño y los principios son:

\section{- Enfoque al cliente \\ - $\quad$ Liderazgo \\ - Participación del personal \\ - $\quad$ Enfoque basado en procesos \\ - $\quad$ Enfoque de sistema para la gestión \\ - $\quad$ Mejora continua \\ - $\quad$ Enfoque basado en hechos para la toma de decisión \\ - Relaciones mutuamente beneficiosas con el proveedor}

Cantú (2004), refiere que un sistema de gestión de calidad tiene dos grandes vías: a) el aseguramiento de la calidad, que abarca la totalidad de los procesos que tienen planeados y organizados y b) el control de la calidad, que es el conjunto de acciones que se utilizan para que los resultados de los procesos de aseguramiento operen según lo planeado, lo cual permite que se logren las especificaciones de calidad.

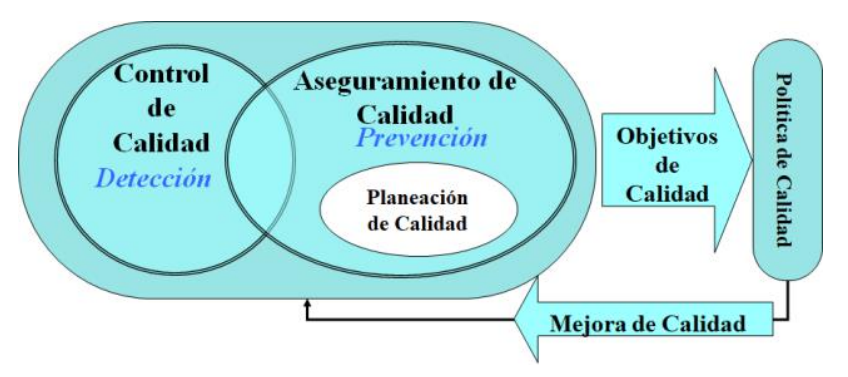

Figura 1 Relación de definiciones de los sistemas de gestión de calidad 
Como se puede observar en la figura, las actividades de la organización que operan en base a sistema de gestión de calidad ISO 9001 están orientadas a cumplimiento de los objetivos y estos a su vez están orientados al cumplimiento de la política de calidad; además, se sabe que la política de calidad incluye compromiso a cumplir los requerimientos de calidad, a la mejora continua y satisfacción de cliente.

\section{Metodología de Investigación}

Esta investigación cuenta con dos etapas. La primera será de carácter documental y la otra en parte de campo. Esta investigación es de tipo correlacional a las causas para describir la relación entre dos o más conceptos variables. También es transversal por lo que se enfocara en un momento determinado de tiempo, Hernández, F. (2006). La investigación considera el análisis de las variables independiente que son los sistemas de gestión de calidad y empresas de manufactura, mientras que el cambio organizacional es la variable dependiente, misma que se busca explicar a partir de la relación que ejercen las independientes; por este esquema aplicado de investigación noexperimental se debe implicar un análisis de alcance transversal y enfoque correlacional. También se considerarán algunas variables intermedias como los procesos clave, objetivos, política de calidad y la asesoría para estar en posición de probar o descarta las hipótesis planteadas.

Para el estudio de campo se aplicará un cuestionario de preguntas cerradas presentadas en forma de afirmaciones o juicios, las cuales nos permitirán evaluar las respuestas de los participantes; debe elegir uno de los puntos "Escala Likert" mostrados en cada pregunta que se presenta en la encuesta, cada respuesta elegida sumará finalmente un total en puntuación obtenida que será contrastada en relación con todos los reactivos de la encuesta. También se pretende sea probabilístico y se validara por un programa de análisis estadístico para con esta información validar la pertinencia de las conclusiones.

\section{Tipo de Investigación}

La investigación se realizará por encuesta, este es el recurso que típicamente utiliza el investigador para colectar y registrar la información y datos de las variables que se han definido como críticas para su trabajo.
El cuestionario es estrecho dependiente de los objetivos de la investigación, de la hipótesis de funcionamiento y del modelo de la investigación.

\section{Métodos Teóricos}

Se plantea un instrumento de medición tipo encuesta. También se harán entrevistas con los directivos, supervisores y operativos para conocer su opinión del cambio organizacional a partir de la implementación del sistema de gestión de calidad. Y se hará un comparativo con empresas que no tengan un sistema de gestión de calidad formal buscando mayor contraste y para poder explicar las similitudes y posibles diferencias.

\section{Metodología de Desarrollo}

La población para el estudio está dada por empresas metalmecánica de tamaño micro y pequeñas que estén certificadas en ISO 9001 o están en vías de certificarse, se seleccionaron por lo siguiente:

Son empresas que por su tamaño las mejoras o las faltas se hacen perceptibles en periodos cortos de tiempo.

- La norma ISO 9001 de sistemas de gestión de calidad son de aplicación general y no son muy pesados en su estructura documental para estas empresas.

De acuerdo a la cantidad de empresas certificadas en ISO 9001 en el municipio de San Juan del Río se calcula el tamaño de muestra para una población estimada de 53 empresas certificadas dato que proporciona CANACINTRA (2013) y asociación de industriales:

Así mismo en este estudio se considerarán las variables y están ubicadas en dos categorías.

\section{Variable principal determinada por la palabra clave}

$\begin{array}{ll}- & \mathrm{X}_{1} \text { : Sistemas de Gestión de Calidad } \\ - & \mathrm{X}_{2}: \text { Empresas certificadas de } \\ \text { manufacturas en el municipio de San } \\ \text { Juan del Río } \\ \text { Y } \\ \text { Y: Cambio Organizacional }\end{array}$

TORRES-TELLO, Marco Antonio, VILLARREAL-ALCALDE, Jorge Gabriel, CHACÓN-PENA, Jair De Jesús y URQUIZA-RESÉNDIZ, Rafael. El impacto del cambio organizacional en las empresas metalmecánicas de San Juan de Río, las cuales cuentan con Sistema de Gestión de Calidad preferentemente certificado. Revista de Desarrollo Económico. 2019 
Sistema de Gestión de Calidad: Se refiere a la Gestión de los procesos de su organización que forman el Sistema de Calidad bajo una norma internacional ISO 9001:2008 así como de la medición del desempeño de los diferentes procesos como: producción, logística, ventas, recursos humanos, ingeniería y de cómo han contribuido en la mejora continua y cambio organizacional.

Empresa Certificada: En este campo se especifica sí la persona que responde la encuesta corresponde a una organización cuyo sistema de gestión de calidad ha sido certificado por un organismo de tercera parte.

Cambio Organizacional: Es el conjunto de variaciones de orden estructural que sufren las organizaciones y que se traducen en un nuevo comportamiento organizacional que permite la mejora de los procesos que forman el sistema de gestión.

\section{Variables Intermedias}

$\begin{array}{ll}- & X_{3}: \text { Asesoría } \\ - & X_{11}: \text { Proceso de los sistemas de Gestión } \\ & \text { de calidad } \\ - & X_{12}: \text { Objetivos de Calidad } \\ - & X_{13}: \text { Política de Calidad }\end{array}$

- Asesoría en Sistemas de Gestión de Calidad, se relaciona con la contribución de la asesoría a la implementación de sistemas de gestión de calidad y el cambio favorable de las organizaciones medido entre otros aspectos a través de la mejora de los indicadores de desempeño de los procesos.

- Los objetivos de calidad se deben establecer de manera que contribuyan de manera efectiva al cumplimiento de la política de calidad.

La política de calidad es una declaración escrita con las intenciones que la alta dirección tiene para con su organización y debe incluir comprimo a la mejora continua y la satisfacción de cliente en base al sistema que a su vez se compone de procesos (ISO 9001:2008).

\section{Relación de variables con las hipótesis}

La selección de herramientas estadísticas y métodos aplicables a la recolección y análisis de datos que permitan validar las hipótesis sin ser limitativo y los cuales podrían ser:

- $\quad$ Antecedentes regionales y globales.

- $\quad$ Estudio dentro del marco teórico en la región y a nivel global.

- $\quad$ Diseño de encuesta y/o cuestionarios.

- Validez de instrumento con Alfa de Crombach.

- Trabajo de campo.

- Validación de las pruebas de hipótesis con apoyo en la estadística como, por ejemplo, t Student, F Fisher, Chi2 entre otras.

\section{Resultados}

Resultados del estudio a través de encuesta por cuestionario.

\section{Interpretación del coeficiente alfa de Cronbach}

Según Oviedo, H., \& Campo-Arias, A. (2005), el valor mínimo aceptable para el coeficiente alfa de Cronbach es 0,70; por debajo de ese valor la consistencia interna de la escala utilizada es baja. Se validó el instrumento (encuesta) a través del alfa de Crombach con 0.77 por arriba del 0.70 que es el mínimo. Se aplicaron un total de 28 encuestas de 37 planeadas que nos da un error de $10.91 \%$ con una confianza del $92 \%$.

Lamentablemente por falta de disposición de algunas empresas a contestar las encuestas los resultados son no probabilísticos, sin embargo, los resultados obtenidos se pueden considerar aplicables a las empresas encuestadas como estudios de caso y quizá por la naturaleza del sector pudieran aplicar a otras en la región.

Los datos en resumen con los índices de centro se muestran en la siguiente tabla: 


\begin{tabular}{|l|r|r|}
\hline & Con Cert & Sin Cert \\
\hline 1.1 & 4.29 & 3.17 \\
\hline 1.2 & 4.57 & 4.00 \\
\hline 1.3 & 4.29 & 3.33 \\
\hline 1.4 & 4.14 & 4.00 \\
\hline 1.5 & 4.71 & 4.17 \\
\hline 1.6 & 4.57 & 3.67 \\
\hline 1.7 & 4.29 & 3.17 \\
\hline 2.1 & 4.43 & 3.17 \\
\hline 2.2 & 4.14 & 3.00 \\
\hline 2.3 & 4.00 & 3.33 \\
\hline 2.4 & 4.14 & 4.00 \\
\hline 2.5 & 4.14 & 3.17 \\
\hline 3.1 & 4.43 & 3.83 \\
\hline 3.2 & 4.29 & 3.67 \\
\hline 3.3 & 4.29 & 3.00 \\
\hline 3.4 & 4.43 & 3.33 \\
\hline 3.5 & 4.29 & 3.17 \\
\hline 3.6 & 4.71 & 3.33 \\
\hline 3.7 & 4.29 & 3.67 \\
\hline Promedio & 4.338 & 3.482 \\
\hline "S" & 0.198 & 0.376 \\
\hline & & \\
\hline
\end{tabular}

Tabla 2

De acuerdo con el estadístico de prueba las percepciones de los encuestados de empresas "certificadas" vs los de empresas "no certificados" son estadísticamente diferentes teniendo una mejor percepción los encuestados de empresas certificadas de 4.3 vs 3.3
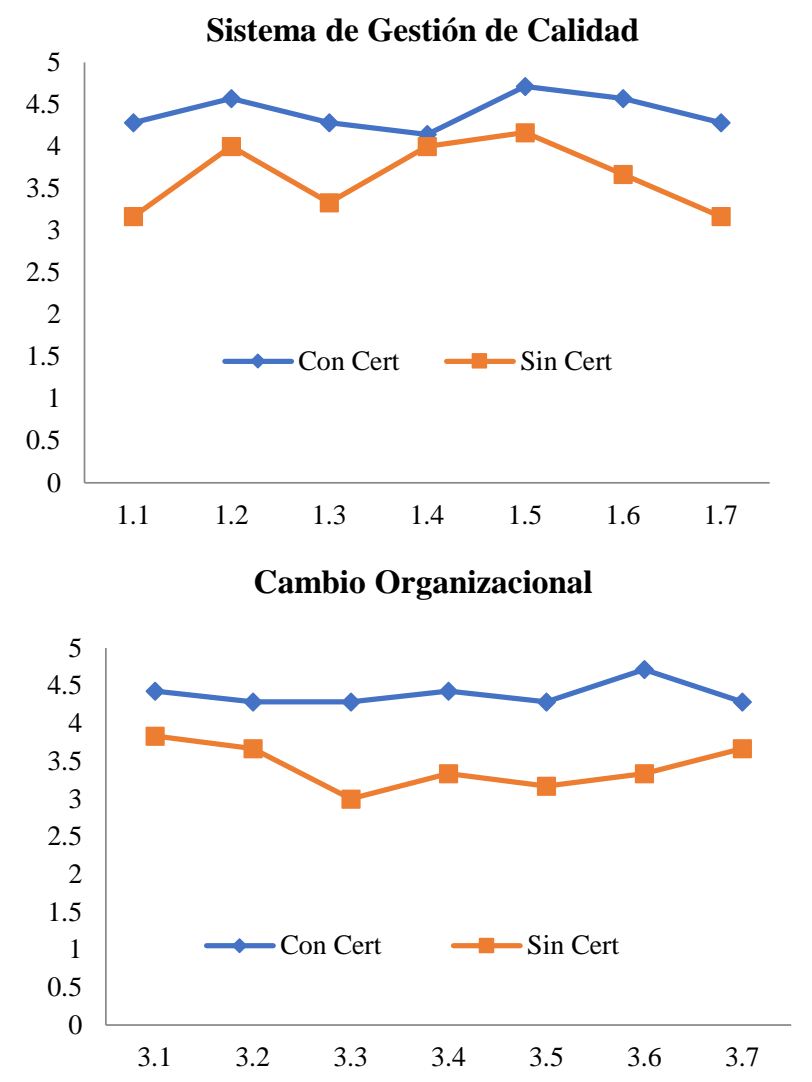

Figura 4 Gráficas poligonales por Item de empresas "Certificadas" VS "No certificadas" usando en Minitab Fuente: Elaboración Propia
Prueba de Hipótesis para un valor objetivo mayor a 4 que es favorable para empresas certificadas. En general todos los encuestados de empresas certificadas coinciden en que tanto los sistemas de gestión de calidad como la asesoría eficiente ayuda al cambio organizacional favourable.

Se rechaza Ho y se acepta $\mathrm{H} 1$, que significa que en general los encuestados de empresas certificadas están de acuerdo que los sistemas de gestión de calidad y la asesoría ayuda a un cambio organizacional favorable a un 95\% de confianza.

Pruebas estadísticas por cada variable para una media mayor que 4

\section{SGC T: Con Cert}

Test of $\mathrm{mu}=4 \mathrm{vs}>4$

95\% Lower

Variable N Mean StDev SE Mean Bound $\mathrm{T}$ P

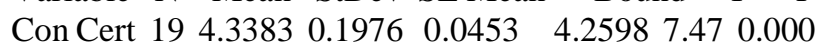
En empresas certificadas los sistemas de gestión de calidad apoyan a un cambio organizacional favorable

\section{ASESORÍA T: ASES_2}

\section{Conclusiones}

Se logro identificar la diferencia de percepciones que tienen los empleados de empresas certificadas y no certificadas en sistemas de gestión de calidad, así como su impacto en los cambios organizacionales favorables.

La percepción de empleados en empresas certificadas en ISO 9001, tienen una mejor percepción de que los sistemas de gestión de calidad y la asesoría ayuda a tener cambios organizacionales favorable con puntuación de acuerdo a la escala de 4.3 vs 3.3 , esta diferencia es estadística utilizando una prueba de medias en minitab 15

Los empleados en empresas no certificadas reconocen igual que los de empresas certificadas que la asesoría es importante para lograr la certificación. Los encuestados de empresas certificadas están de acuerdo con ponderación mayor a 4 que la los sistemas de gestión de calidad y la asesoría ayuda a un cambio organizacional favorable a un $95 \%$ de confianza. 
Los empleados de empresas no certificadas en sistemas de gestión de calidad con promedio de ponderación de 3 no están seguros que los sistemas de gestión de calidad generen cambios organizacionales positivos.

De acuerdo con la investigación realizada, se puede presumir que los procesos de los sistemas de gestión de calidad tienen un impacto a la eficacia de los sistemas y a la competitividad por lo que resulta interesante más adelante seguir otra línea de investigación para identificar los factores clave que tienen mayor impacto a la competitividad y cambio organizacional.

\section{Referencias}

Audirac, C. (1995). El desarrollo Organizacional. Editorial Trillas, México 1995.

Daft, Richard L. (2004). Administración. Sexta Edición. México: Thomson.

Daft, Richard L. (2010). Introducción a la Administración. (6 $6^{\mathrm{a}}$ ed.) México: Cengage.

Davis, K. \& Newstrom, J. (2001), Comportamiento de las organizaciones, McGraw Hill. México 2001

Deming, W. E. (1989). Calidad, productividad y competitividad. La salida de la crisis. .Madrid: Díaz de Santos.

Hernández, R. Fernández, C. y Baptista, P. (2006). Metodología de la Investigación, México: McGraw-Hill

Feigenbaum. A. V. (1986). Control total de Calidad. México: Continental.

Huang, S., Chen, J., Ye, W., \& Wang, K. (2019). The effect of external partner heterogeneity on open innovation: the moderating role of the technological regime. Technology Analysis \& Strategic Management, 31(5), 593-605.

Huamán, J., Llontop, J., Raymundo, C., \& Dominguez, F. (2019, September). Production Management Model Based on Lean Manufacturing Focused on the Human Factor to Improve Productivity of Small Businesses in the Metalworking Sector. In International Conference on Human Systems Engineering and Design: Future Trends and Applications (pp. 847-853). Springer, Cham.
Noor, A., Mahmudah, S., \& Zebua, M. (2019). The Role of Political Will and Organizational Culture toward Iron and Steel Company Competition.

Oliveira, E., Tereso, A. P., \& Santos, C. (2019). Application of a risk management methodology in industrial projects: a case study in the metalworking sector.

Pucheta-Martínez, M. C., Bel-Oms, I., \& Rodrigues, L. L. (2019). The engagement of auditors in the reporting of corporate social responsibility information. Corporate Social Responsibility and Environmental Management, 26(1), 46-56. 